\title{
Antimicrobial and Anticancer Activities of Actinomycetes Isolated from Egyptian Soils
}

\author{
Tarek R. Elsayed ${ }^{1}$, Diana F. Galil ${ }^{1}$, Mohammed Z. Sedik ${ }^{1}$, \\ Hazem M.M. Hassan ${ }^{2,3}$, Michael R. Gohar ${ }^{1}$ and Mahmoud W. Sadik ${ }^{1 *}$ \\ ${ }^{1}$ Department of Microbiology, ${ }^{2}$ Department of Biochemistry, Faculty of Agriculture, \\ Cairo University, Giza, Egypt \\ ${ }^{3}$ Department of Clinical Laboratory Sciences, College of Applied Medical Sciences, \\ Shaqra University, Saudi Arabia \\ *Corresponding author
}

\section{A B S T R A C T}

\section{Keywords}

Actinomycetes; secondary metabolites; Antimicrobial, Anticancer

\section{Article Info}

Accepted: 15 August 2020 Available Online: 10 September 2020
Actinomycetes have got considerable attention worldwide due to the production of many useful bioactive metabolites. In the present study, the antimicrobial potential of novel Actinomycetes has been evaluated by the initial screening of seven rhizosphere samples in Egypt. The primary and secondary screening were performed against Bacillus cereus (ATCC33018), and E.coli O157 (ATCC93111) as model strains of Gram positive and Gram negative species, respectively. GCMS analysis of most active Actinomycetes extracted secondary metabolites was studied. The major compounds identified by GC-MS analysis include 2, 4-di-tertbutylphenol, 3-chloropropionic acid, heptadecyl ester, 1,2-benzenedicarboxylic acid,3-nitro, benzenepropanoic acid,3,5-bis(1,1 dimethylethyl)-4-hydroxy-, octadecyl ester, 1-monopalmitin,2TMS derivative, 1-docosene, 1-nonadecene and 1-nonacosene.

\section{Introduction}

Actinomycetes categorized as Gram-positive filamentous bacteria with fungal morphology. They are characterized under group of phylum Actinobacteria (1). They were distributed in different ecosystems especially soil ecosystem. They play an important role in different environmental activities such as recycling of agricultural and industrial wastes by decomposing complex organic polymeric structures in agro-industrial wastes through active interactions with fungi (2).

Actinomycetes have an important role in agroindusterial and biomedical areas such as composting production, antimicrobial products, antioxidants, enzyme inhibitors and antitumor products $(3,4,5,6,7)$. Due to the emergence of multidrug-resistant microorganisms to almost all available antibiotics, many researchers are focused now 
on discovering novel antimicrobials from many natural resources such as those produced by Actinomycetes especially those isolated from many undiscoverable or poorly explored environments (8). Different reports were considered that Egyptian soil is poorly investigated source for actinobacteria, $(9,10$, 11, 12). Also, thousands of bioactive medical compounds have been discovered from Actinomycetes and characterized in treatment of wide range of diseases in human, veterinary, and agriculture sectors $(13,14$, $15)$.

Hence, the Actinomycetes are considered to be the most potent source for the production of secondary active metabolites such as antibiotics, and other bioactive compounds. It is well established that each Actinomycete strain has probably genetic potential ability to produce 10-20 secondary metabolites $(16,17$, 18). Actinomycetes produced many antibacterial agents such as tetracyclines and antifungal agents such as amphotericin, and anticancer drugs exemplified by Adriamycin and the immunosuppressant tacrolimus (18). Actinomycetes has been reported to contribute nearly $70 \%$ of metabolites described under actinobacteria (19). All studies continue to discover natural antimicrobial agents from actinomycetes to be useful sources of novel secondary metabolites and study their applications as anti-bacterial, antifungal and anticancer agents, or other pharmaceutically useful compounds (20).

In these perspectives, the current study aimed to isolate and characterize different Actinomycetes from soil niches in the Giza governorate, Egypt.

Actinomycetes were screened for their capabilities to produce antimicrobial secondary active metabolites. The bioactive compound from the most potent isolates was characterized using GC-MS analysis.

\section{Materials and Methods}

\section{Sampling and isolation of actinomycetes}

Soil samples were collected from the rhizosphere of different crops. A total of 7 rhizosphere samples were collected from (Hordeum vulgare, Allium cepe, Trifolium, Brassica oleracea, Triticum aestivum, Solanum lycopersicum, and Pelargonium graveolens) crop soil samples (Table 1). Serial dilutions were prepared from rhizosphere samples and a dilution plate method was used to isolate Actinomycetes on starch-nitrate agar plates (21). The plates were incubated for 7 days at $30^{\circ} \mathrm{C}$. Colonies with the typical Actinomycetes morphology were picked and checked for purity by repeated sub-culturing. Pure cultures were maintained on Luria-Bertani (LB) broth supplemented with $20 \%$ glycerol at -20 for further use.

\section{Screening of actinomycetes for antimicrobial activity}

Actinomycetes isolates were screened against various test organisms (E.coli O157 ATCC and Bacillus cereus ATCC) by dual culture plate assay. The effect of Actinomycetes was tested by placing a six mm diameter disk of 7 days old fully grown Actinomycetes on starch nitrate agar plates seeded with $10 \%$ of each bacterial pathogen. The diameter of inhibition zones was recorded after $24 \mathrm{~h}$ of incubation at $37{ }^{\circ} \mathrm{C}(22)$.

\section{The effect of secondary metabolites on cancer cells}

Cell cytotoxicity effects of Actinomycetes secondary active metabolites were estimated on human lung cancer cell line (A549) using neutral red uptake assay according to (23). Cells were grown as a monolayer culture in RPMI 1640 medium (10\% fetal bovine serum, $1 \mathrm{mM}$ sodium pyruvate and $100 \mathrm{U} / \mathrm{L}$ of 
penicillin/streptomycin) and incubated at $37 \mathrm{C}^{\circ}$ in a $5 \%$ of $\mathrm{CO}_{2}$ atmosphere. Cell lines (100 $\mu \mathrm{L})$ were seeded in 96 well plates at a concentration of $5 \times 10^{3}$ cells $/ \mathrm{mL}$, for $24 \mathrm{~h}$. After that, the culture medium was replaced with $100 \mu \mathrm{L}$ serum-free medium containing various concentrations $(25,50,100,150,200$, $250,300,350,400,450$ and $500 \mu \mathrm{g} / \mathrm{mL}$ ) of Actinomycete extracts at $24 \mathrm{~h}$ and $48 \mathrm{~h}$. Later, the medium was refreshed with $100 \mu \mathrm{L}$ of serum free medium (RPMI 1640) and $20 \mu \mathrm{L}$ of MTT $(5 \mu \mathrm{g} / \mathrm{mL}$ of $(3,4,5$-dimethylthiazol2yl)-2, 5-diphenyltetrazo liumbromide).

The 96 well plates were incubated for $3 \mathrm{~h}$ in dark and the developed color was measured with ELISA reader at $570 \mathrm{~nm}$. Triplicates were maintained for each treatment. Inhibitory concentration (IC50) values were directly determined by linear regression analysis with office XP (SDAS) software.

\section{Results and Discussion}

\section{Isolation of Actinomycetes}

A total of 37 Actinomycetes were isolated from the rhizosphere of different crops. After purification on starch nitrate agar (SNA) medium bacterial isolates were placed on LB medium supplemented with $20 \%$ glycerol and preserved at -20 .

\section{Antimicrobial activities tests}

A total of 8 Actinomycetes isolates from 37 isolates showed antimicrobial activity against at least one of the tested bacterial pathogens such as E. coli O157 (ATCC 9311) and Bacillus cereus (ATCC33018). The diameters of inhibition zones are present in (Table 2) comparing to positive control. Maximum activity was recorded as $11 \pm 1 \mathrm{~mm}$ against Bacillus cereus by isolate AC-2. Maximum activity was recorded against E. coli $O 157$ $(11 \pm 2 \mathrm{~mm})$ by isolate AC-7.

\section{Anticancer effect of extracted bioactive compounds}

The effect of the Actinomycetes AC-6 secondary bioactive metabolite was tested against Adenocarcinoma of Human Lung cancer cell line (A549). As clearly shown in Table (3) the effect of secondary metabolites of actinomycetes strains on Human Lung cancer cell line (A549) exhibited very low activity as anticancer agent (LC $50=1122$ $\mu \mathrm{g} / \mathrm{ml})$.

\section{Identification of potentially bioactive compounds by GC-MS}

The chemical identification of the bioactive compounds present in the ethyl acetate extracts of Actinomycetes AC-6, AC-7 and AC-8 was performed by GC-MS based on the retention time, peak areas, molecular weight and molecular formula. The GC-MS obtained for the extracts tested showed defined signal patterns characteristic of each type of extract as indicated by peaks.

The major compounds identified by GC-MS analysis (Figure 1 and Table 4) in ethyl acetate extracts of Actinomycetes (AC-6, AC7 and AC-8) were 2,4-di-tert-butylphenol, 3chloropropionic acid, heptadecyl ester, 1,2benzenedicarboxylic acid, 3-nitro, benzene propanoic acid, 3,5-bis(1,1 dimethyl ethyl)-4hydroxy-,octadecyl ester, 1-monopalmitin, 2TMS derivative, 1-docosene, 1-nonadecene and 1-nonacosene.

Twenty four compounds were identified by GC-MS in Actinomycetes AC-6 secondary metabolites, The most predominant major compounds were 2,4-di-tert-butylphenol (20.19\%), 3-chloropropionic acid heptadecyl ester (13.40\%), 1,2-benzenedicarboxylic acid, 3-nitro $(6.87 \%)$, tributylacetylcitrate $(5.90 \%)$ and squalene $(4.48 \%)$ and the minor compounds were 1-monopalmitin, 2TMS 
derivative $(2.64 \%)$, 1-docosene $(2.61 \%), 1-$ nonadecene $(2.57 \%)$, benzenepropanoic acid, 3,5-bis(1,1 dimethylethyl)-4-hydroxy-, octadecyl ester $(2.15 \%)$, isopropyl myristate (2.10\%), 3-eicosene, (E)- (1.92\%), 2propenoic acid, 3-(4-methoxyphenyl)-, 2ethylhexyl ester (1.48\%), 1-nonacosene (1.40\%), dotriacontane (1.28\%), 1-(4isopropylphenyl)-2-Methylpropyl acetate $(1.13 \%)$, docosane (1.12\%) and 1,3benzenedicarboxylic acid, bis (2-ethylhexyl) ester $(1.03 \%)$. The remaining compounds were present in less than $1 \%$. On the other hand, thirteen active compounds were identified by GC-MS in Actinomycetes AC-7 secondary metabolites, the active major compounds were 2,4-di-tert-butylphenol (32.66\%), 3-chloropropionic acid, heptadecyl ester (19.93\%), benzenepropanoic acid, 3,5bis(1,1 dimethylethyl)-4-hydroxy-, octadecyl ester $(5.54 \%)$ and 1-monopalmitin, 2TMS derivative $(4.28 \%)$ and the minor compounds were 1-docosene (3.99\%), 1-nonadecene (3.38\%), 3-eicosene, (E)- (3.35\%), 1,2benzenedicarboxylic acid, 3-nitro (3.28\%), 1nonacosene $(2.76 \%)$, 9-octadecenamide (1.51\%), oxiraneundecanoic acid, 3-pentyl-, methyl ester, trans (1.31\%), oleic acid, 3(Octadecyloxy) propyl ester (1.09\%) and isochipane B $(0.85 \%)$. In addition, twelve active compounds were found in Actinomycetes AC-8 secondary metabolites by GC-MS analysis. The prevailing compounds were 2,4-di-tert-butylphenol (32.42\%), 3-chloropropionic acid, heptadecyl ester (17.51\%), 1-docosene (4.39\%), 1hexadecanol $(3.80 \%)$, 1-nonadecene $(3.73 \%)$, 1-monopalmitin, 2TMS derivative (3.20\%), 1nonacosene $(2.71 \%), 1,2$-benzenedicarboxylic acid, 3-nitro (2.59\%), 9-octadecenamide (2.18\%), benzenepropanoic acid,3,5-bis $(1,1$ dimethylethyl)-4-hydroxy-, octadecyl ester $(2.11 \%)$ and dotriacontane $(0.97 \%)$.The biological functions of these compounds were identified in the ethyl acetate extracts of Actinomycetes AC-6, AC-7 and AC-8 as shown in Table 4.

The present study aimed to determine the effect of Actinomycetes secondary metabolites on different pathogen by inducing the antimicrobial activity in different pathogens. In addition to its anticancer activity.

Table.1 Actinomycetes isolated from the rhizosphere soil of different crops

\begin{tabular}{|l|l|l|}
\hline Strain & Crop name & Source \\
\hline AC-1 & Hordeum vulgare & $\begin{array}{l}\text { Faculty Agriculture, Cairo } \\
\text { University }\end{array}$ \\
\hline AC-2 & Triticum aestivum & $\begin{array}{l}\text { Faculty Agriculture, Cairo } \\
\text { University }\end{array}$ \\
\hline AC-3 & Triticum aestivum & $\begin{array}{l}\text { Faculty Agriculture, Cairo } \\
\text { University }\end{array}$ \\
\hline AC-4 & Allium cepa & $\begin{array}{l}\text { Faculty Agriculture, Cairo } \\
\text { University }\end{array}$ \\
\hline AC-5 & Brassica olevacea & Eastern Province, Egypt \\
\hline AC-6 & Trifolium & Eastern Province, Egypt \\
\hline AC-7 & Pelargonium graveolens & Nile Delta, Egypt \\
\hline AC-8 & Solanum lycopersicum & Nile Delta, Egypt \\
\hline
\end{tabular}


Table.2 Antimicrobial activities of isolated Actinomycetes

\begin{tabular}{|l|l|l|}
\hline Actinomycetes & Bacillus cereus & E. coli O157 \\
\hline Positive control & $10.0 \pm 2.0$ & $11.0 \pm 1.0$ \\
\hline AC-1 & - & - \\
\hline AC-2 & $11.0 \pm 1.0$ & - \\
\hline AC-3 & $8.0 \pm 2.0$ & $8.0 \pm 1.0$ \\
\hline AC-4 & $8.0 \pm 2.0$ & $9.0 \pm 1.0$ \\
\hline AC-5 & $9.0 \pm 2.0$ & $11.0 \pm 2.0$ \\
\hline AC-6 & $9.0 \pm 2.0$ & $8.0 \pm 1.0$ \\
\hline AC-7 & $7.0 \pm 1.0$ & - \\
\hline AC- 8 & - & $8.0 \pm 1.0$ \\
\hline
\end{tabular}

Inhibition zones are represented in $\mathrm{mm}$, the diameter of Actinomycetes disk is $6 \mathrm{~mm}$

Table.3 Anticancer effect of extracted bioactive compounds of strain AC-6

\begin{tabular}{|l|l|}
\hline $\begin{array}{l}\text { Concentration } \\
(\boldsymbol{\mu} \mathbf{g} / \mathbf{m l})\end{array}$ & Viability \% \\
\cline { 2 - 2 } AC-6 \\
\hline 50 & 97 \\
\hline 25 & 99.3 \\
\hline IC 50 & 99.6 \\
\hline
\end{tabular}

Table.4 GC-MS analysis of the ethyl acetate extracts of AC-6, Ac-7and Ac-8)

\begin{tabular}{|c|c|c|c|c|c|c|c|}
\hline \multirow[t]{2}{*}{ RT } & \multicolumn{3}{|c|}{ Percentage area } & \multirow[t]{2}{*}{ Compound name } & \multirow{2}{*}{$\begin{array}{l}\text { Molecular } \\
\text { formula }\end{array}$} & \multirow{2}{*}{$\begin{array}{l}\text { Molecular } \\
\text { weight }\end{array}$} & \multirow[t]{2}{*}{ Proposed function } \\
\hline & AC-6 & AC-7 & AC-8 & & & & \\
\hline 14.58 & - & - & 3.8 & 1-Hexadecanol & $\mathrm{C}_{14} \mathrm{H}_{34} \mathrm{O}$ & 242 & Antibacterial (35) \\
\hline 14.62 & 1.92 & 3.35 & - & 3-Eicosene, (E)- & $\mathrm{C}_{20} \mathrm{H}_{40}$ & 280 & $\begin{array}{l}\text { Antimicrobial, Antihyperglycemic, } \\
\text { Cytotoxic Activity, Antioxidant, } \\
\text { Insecticidal activity (36) }\end{array}$ \\
\hline 15.76 & 20.19 & 32.66 & 32.42 & $\begin{array}{c}\text { Phenol, } \\
\text { 2,4-Bis }(1,1- \\
\text { dimethyl ethyl) }\end{array}$ & $\mathrm{C}_{14} \mathrm{H}_{22} \mathrm{O}$ & 206 & $\begin{array}{c}\text { Antioxidant, Analgesic, Anticancer } \\
\text { (37) }\end{array}$ \\
\hline 17.96 & 2.61 & 3.99 & 4.39 & 1-Docosene & $\mathrm{C}_{22} \mathrm{H}_{44}$ & 308 & $\begin{array}{c}\text { Antibacterial, } \\
\text { Anti-inflammatory (38) }\end{array}$ \\
\hline 18.37 & 1.13 & - & - & $\begin{array}{c}1-(4- \\
\text { IsoPropylPhenyl)2- } \\
\text { Met-Hylpropyl } \\
\text { Acetate }\end{array}$ & $\mathrm{C}_{15} \mathrm{H}_{22} \mathrm{O}_{2}$ & 234 & $\begin{array}{l}\text { Anti-inflammatory, antiheistamanial } \\
\text { and antitrypanosoma (39) }\end{array}$ \\
\hline 19.38 & 0.05 & - & - & Pentacosane & $\mathrm{C}_{25} \mathrm{H}_{52}$ & 352 & $\begin{array}{l}\text { Major component of essential } \\
\text { oils (40) }\end{array}$ \\
\hline 19.47 & 2.10 & - & - & $\begin{array}{c}\text { Isopropyl } \\
\text { Tetradecanoate }\end{array}$ & $\mathrm{C}_{17} \mathrm{H}_{34} \mathrm{O}_{2}$ & 270 & Anti-inflammatory (41) \\
\hline 20.84 & 0.76 & - & - & $\begin{array}{l}\text { Methoxyacetic } \\
\text { acid, 2-tetradecyl } \\
\text { Ester }\end{array}$ & $\mathrm{C}_{17} \mathrm{H}_{34} \mathrm{O}_{3}$ & 286 & Anti-microbial (35) \\
\hline 21.05 & 2.57 & - & 3.73 & 1-Docosene & $\mathrm{C}_{22} \mathrm{H}_{44}$ & 308 & Antibacterial (38) \\
\hline
\end{tabular}




\begin{tabular}{|c|c|c|c|c|c|c|c|}
\hline 21.06 & - & 3.38 & - & 2-Hexadecanol & $\mathrm{C}_{16} \mathrm{H}_{34} \mathrm{O}$ & 242 & Antimicrobial activity (38) \\
\hline 21.41 & 0.59 & - & - & $\begin{array}{l}\text { Tetradecanoic } \\
\text { Acid, 12-Methyl- } \\
\text { Ester }\end{array}$ & $\mathrm{C}_{16} \mathrm{H}_{32} \mathrm{O}_{2}$ & 256 & $\begin{array}{l}\text { Antioxidant, cancer preventive, } \\
\text { Nematicide, lubricant and } \\
\text { Hypocholesterolemic (42) }\end{array}$ \\
\hline 21.58 & - & 1.31 & - & $\begin{array}{l}\text { Oxiraneundecanoic } \\
\text { acid, 3-Pentyl- } \\
\text { Methyl Ester }\end{array}$ & $\mathrm{C}_{1}{ }_{9} \mathrm{H}_{36} \mathrm{O}_{3}$ & 312 & Anti-oxidant $(43)$ \\
\hline 22.08 & 0.73 & & & 2-Hexadecanol & $\mathrm{C}_{16} \mathrm{H}_{34} \mathrm{O}$ & 242 & Anti-microbial (38) \\
\hline 27.30 & 1.48 & & & $\begin{array}{l}\text { 2-Propenoic acid, } \\
\text { 3-(4- } \\
\text { methoxyphenyl)-, } \\
\text { 2-ethylhexyl } \\
\text { ester }\end{array}$ & $\mathrm{C}_{18} \mathrm{H}_{26} \mathrm{O}_{3}$ & 290 & Anti- inflammatory(41) \\
\hline 27.53 & 1.28 & & 0.97 & $\begin{array}{c}\text { Ethanol, 2- } \\
\text { (octadecyloxy) }\end{array}$ & $\mathrm{C}_{20} \mathrm{H}_{42} \mathrm{O}_{2}$ & 314 & Antimicrobial (50) \\
\hline 27.77 & 5.9 & & & $\begin{array}{l}\text { TriButylAcetylcitr } \\
\text { ate }\end{array}$ & $\mathrm{C}_{20} \mathrm{H}_{34} \mathrm{O}_{8}$ & 402 & no activity \\
\hline 27.98 & & 0.89 & & $\begin{array}{l}\text { 13-Methyl.13- } \\
\text { Carpinalpodo carp- } \\
\text { 7EN 3B 3BXIDiol } \\
\text { CARP-7-EN- } \\
\text { 3B,X-DIOL }\end{array}$ & $\mathrm{C}_{19} \mathrm{H}_{30} \mathrm{O}_{3}$ & 306 & no activity \\
\hline 28.72 & 2.27 & 1.67 & 2.04 & Heptacosane & $\mathrm{C}_{27} \mathrm{H}_{56}$ & 380 & antibacterial(44) \\
\hline 28.95 & 2.64 & 4.28 & 3.2 & $\begin{array}{c}\text { 1-Monopalmitin, } \\
\text { 2TMS derivative } \\
\text { 2,3-Bis } \\
\text { ((TriMethylSilylL) } \\
\text { OXY) Propy Ester }\end{array}$ & $\begin{array}{c}\mathrm{C}_{25} \mathrm{H}_{54} \mathrm{O}_{4} \mathrm{Si} \\
2\end{array}$ & 474 & no activity \\
\hline 28.97 & & & 0.86 & 1-Heptatriacotanol & $\mathrm{C}_{37} \mathrm{H}_{76} \mathrm{O}$ & 536 & Antimicrobial (35) \\
\hline 29.33 & 1.54 & & & $\begin{array}{l}\text { 2-Propenoic acid, } \\
\text { 3-(4- } \\
\text { Methoxyphenyl)-, } \\
\text { 2EthylHexyl Ester }\end{array}$ & $\mathrm{C}_{18} \mathrm{H}_{26} \mathrm{O}_{3}$ & 290 & Anti- inflammatory (41) \\
\hline 29.68 & - & - & 2.18 & 9-Octadecenamide & $\mathrm{C}_{18} \mathrm{H}_{35} \mathrm{NO}$ & 281 & $\begin{array}{c}\text { Anti-oxidant and anti-inflammatory } \\
\text { effect (45) }\end{array}$ \\
\hline 29.69 & & 1.51 & - & 9-Octadecenamide & $\mathrm{C}_{18} \mathrm{H}_{35} \mathrm{NO}$ & 281 & $\begin{array}{c}\text { Anti-oxidant and anti-inflammatory } \\
\text { effect (45) }\end{array}$ \\
\hline 29.88 & 1.89 & 0.74 & 0.97 & 1-Docosene & $\mathrm{C}_{22} \mathrm{H}_{46}$ & 310 & Antibacterial (38) \\
\hline 30.99 & 2 & 1.47 & 1.62 & Heptacosane & $\mathrm{C}_{27} \mathrm{H}_{56}$ & 380 & Antibacterial (44) \\
\hline 31.26 & 6.87 & 3.28 & 2.59 & $\begin{array}{c}1,2- \\
\text { BenzenedicaRboxy } \\
\text { lic Acid, 3-Nitro }\end{array}$ & $\mathrm{C}_{8} \mathrm{H}_{5} \mathrm{NO}_{6}$ & 211 & Antibacterial and antioxidant (38) \\
\hline 31.52 & - & 1.09 & - & $\begin{array}{c}\text { Oleic Acid, } 3- \\
\text { (Octadecyloxy)pro } \\
\text { pyl Ester }\end{array}$ & $\mathrm{C}_{39} \mathrm{H}_{76} \mathrm{O}_{3}$ & 592 & Antifungal (46) \\
\hline 32.08 & 1.21 & 0.78 & 0.78 & Ceidoniol, Deoxy & $\mathrm{C}_{29} \mathrm{H}_{60}$ & 408 & $\begin{array}{l}\text { Anti-inflammatory and Anti-cancer } \\
\text { (47) }\end{array}$ \\
\hline 32.62 & 1.03 & - & - & $\begin{array}{c}1,3- \\
\text { Benzenedicarboxyl } \\
\text { ic Acid,Bis }(2-\end{array}$ & $\mathrm{C}_{24} \mathrm{H}_{38} \mathrm{O}_{4}$ & 390 & $\begin{array}{c}\text { Aantibacterial., Antioxidant and } \\
\text { antiviral (38) }\end{array}$ \\
\hline
\end{tabular}




\begin{tabular}{|l|l|l|l|l|l|l|l|}
\hline $\mathbf{3 2 . 8 1}$ & 4.48 & - & - & EethylHexyl) Ester & & & \\
& & & Squalene & $\mathrm{C}_{30} \mathrm{H}_{50}$ & 410 & $\begin{array}{c}\text { Antibacterial, antioxidant, } \\
\text { antitumor, cancer preventive, } \\
\text { immunostimulant (49) }\end{array}$ \\
\hline $\mathbf{3 3 . 1 3}$ & 1.12 & - & - & Docosane & $\mathrm{C}_{22} \mathrm{H}_{46}$ & 310 & Antibacterial (42) \\
\hline $\mathbf{3 3 . 1 4}$ & - & 0.84 & 0.89 & $\begin{array}{c}\text { Isochipane B } \\
\mathrm{C}_{19} \mathrm{H}_{22} \mathrm{O}_{6}\end{array}$ & 346 & \begin{tabular}{c} 
Antibacterial (48) \\
\hline $\mathbf{3 4 . 1 7}$
\end{tabular} \\
\hline 0.67 & - & - & $\begin{array}{c}\text { Octadecane, 3- } \\
\text { Ethyl-5-(2- } \\
\text { EthylButyl)- }\end{array}$ & $\mathrm{C}_{26} \mathrm{H}_{54}$ & 366 & $\begin{array}{c}\text { anti-inflammatory, analgesic } \\
\text { antipyretic, antimicrobial, } \\
\text { antioxidant, antitumor and anti- } \\
\text { diabetic activities (42) }\end{array}$ \\
\hline $\mathbf{3 5 . 1 9}$ & 0.57 & - & - & Isochipane B $\% 2<$ & $\mathrm{C}_{19} \mathrm{H}_{26} \mathrm{O}_{6}$ & 350 & Antibacterial (48) \\
\hline $\mathbf{3 5 . 9 3}$ & 0.75 & - & - & Oleic Acid & $\mathrm{C}_{18} \mathrm{H}_{34} \mathrm{O}_{2}$ & 282 & Anti-inflammatory(38) \\
\hline
\end{tabular}

Fig.1 GC-MS analysis of the ethyl acetate extract of AC-6, AC-7 and AC-8

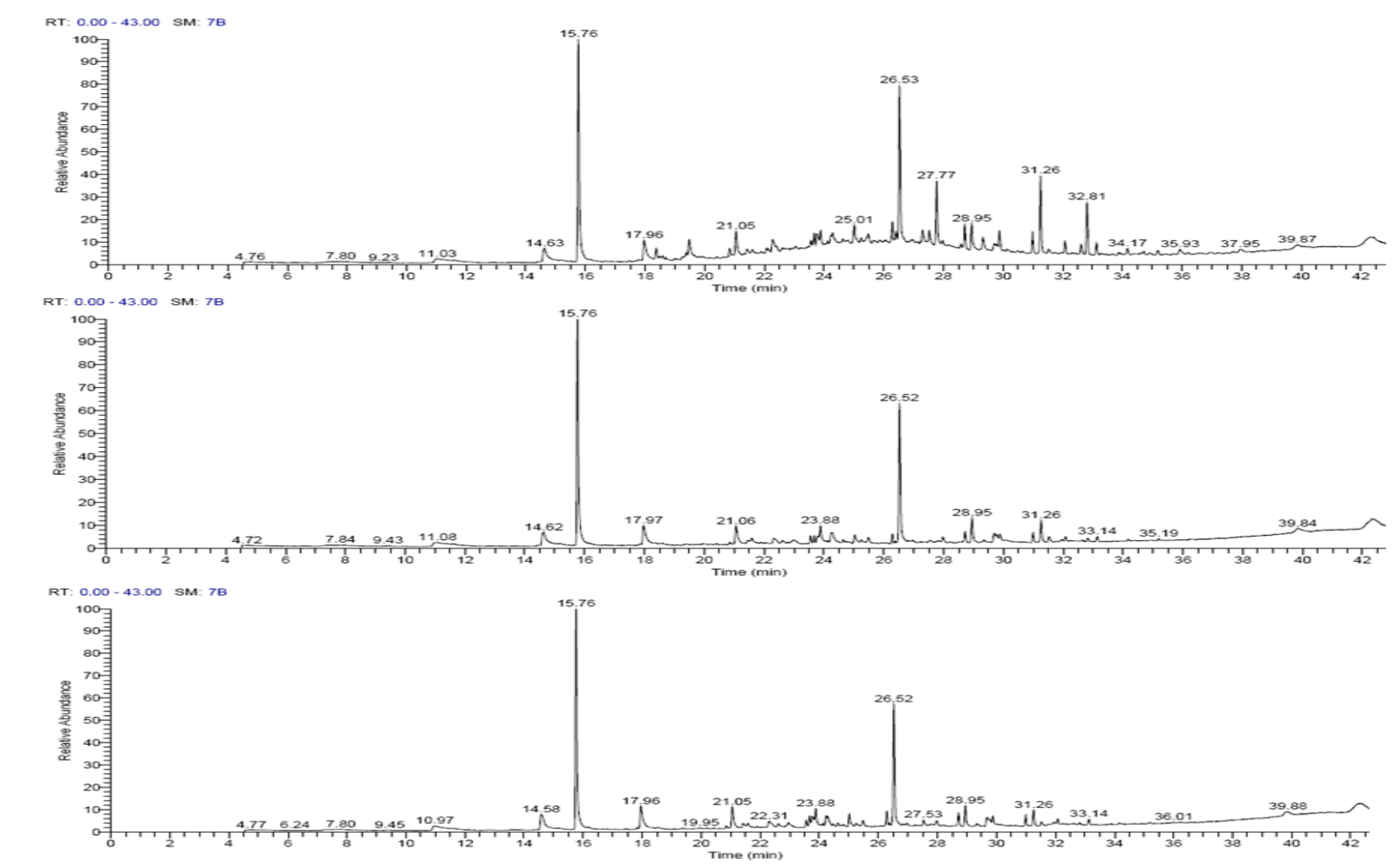

The Actinomycetes were tested against Bacillus cereus, Escherichia coli O157, IsolateAC-2 strain showed antimicrobial activity against $B$. cereus. Isolate AC-5 showed the highest inhibition zone against E.coli $\mathrm{O} 157$.

The results of GC-MS analysis revealed that the ethyl acetate extracts of Actinomycetes metabolites (AC-6, AC-7 and AC-8) contain various constituents like essential oils, fatty acids, esters, alcohols, phenols, alkanes, steroids, and terpenes such as 2,4-di-tertbutylphenol, 3-chloropropionic acid, heptadecyl ester, 1,2-benzenedicarboxylic acid, 3-nitro, benzenepropanoic acid, 3,5bis(1,1 dimethylethyl)-4-hydroxy-,octadecyl ester, 1-monopalmitin, 2TMS derivative, 1docosene, 1-nonadecene, 1-nonacosene and eicosene (Table 4). These active compounds 
possesses different biological activities such as antimicrobial, antibacterial, antifungal, antioxidant, antitumor, anti-hyperglycemic, analgesic, anti-inflammatory, antileishmanial, antitrypanosoma, antispasmodic, antituberculosis, antipyretic, nematicide and lubricant $(35,36,37,38,39,41,42,43,45$, $46,48,51,52,53)$. The obtained results were supported by (24) who reported that secondary metabolism is of special interest in Streptomyces, that occupies a high position in the developmental hierarchy of bacteria due to their advanced morphology and physiology. Streptomycetes have evolved a plethora of biosynthetic pathways to produce various secondary metabolites, especially signal molecules, or antibiotics. These compounds provide the organism with a competitive advantage, protection from unfavorable living conditions and/or facilitate interspecies interactions and have an antimicrobial, antioxidant and anticancer effect (25).

According to (26) the compounds generated by AUDT 217, thirteen compounds have an antimicrobial or antitumor characteristic. This study in parallel with results of (50).who indicated that marine actinomycetes are efficient producers of new secondary metabolites that show a range of biological activities including antibacterial, antifungal, anticancer, insecticidal and enzyme inhibition. Bioactive compounds from marine actinomycetes possess distinct chemical structures that may form the basis for synthesis of new drugs that could be used to combat resistant pathogens.

Studies of $(16,28)$ confirmed that some genes share for biosynthesis of Actinomycetes secondary active metabolites in their clustering and regulation. The chemical structure has so far been elucidated in less than 30 percent of the compounds, belonging to the following groups of natural substances: polyketides, pyrones, peptides, siderophores, $\gamma$-butyrolactones, butenolides, furans, terpenoids, fatty acids, oligopyrroles, and deoxysugars as indicated in our study (24). The remaining 70 percent are called "cryptic compounds" as they are not produced at standard laboratory conditions $(16,29,30,28$, 24). To activate these cryptic pathways, Actinomycetes are cultivated under nonstandard physical and nutritional conditions or co-cultured with other microorganisms (31). Genetic manipulations within the genes (32)or the transfer of the whole biosynthetic gene cluster into a heterologous producer (33, 28)are also commonly used strategies. The successful activation of the biosynthetic pathways often leads to biosynthesis of previously unknown compounds $(27,30,34$, 28).

In conclusion the actinomycetes, especially Streptomycetes, still an important source for bioactive compounds that are used for treating infection diseases, cancer, and many other diseases. The derivative of bioactive metabolites produced by Actinomycetes isolated from Giza Governorate, Egypt, demonstrated obvious inhibitory effects against both Gram-positive and Gram negative bacteria.

\section{Acknowledgements}

The paper and he research behind it would not have been possible without the exceptional support of our supervisor, Meshal Gohar, his enthusiasm, knowledge and exacting attention to detail have been an inspiration and kept our work on to the final form.

\section{References}

1. Dilip, C. V., Mulaje, S., and Mohalkar, R. (2013). A review of actinomycetes and their biotechnological application. Int. J. Pharm. Sci. Res. 4, 1730-1742. 
2. Goodfellow, M., and Williams, S. (1983). Ecology of actinomycetes. Annu. Rev. Microbiol. 37, 189-216. DOI: 10.1146/annurev.mi.37.100183.001201

3. Sacramento, D. R., Coelho, R. R. R., Wigg, M. D., Toledo Luna Linhares, L. F. D., Matos dos Santos, M. G., AzevedoSoaresSemêdo, L. T. D., et al., (2004). Antimicrobial and Antiviral Activities Of An Actinomycete (Streptomyces Sp.) Isolated From Brazilian Tropical Forest Soil. World J. Microbiol. Biotechnol. 20, 225-229. Doi: 10.1023/B: Wibi.0000023824.20673.2f

4. Atta, H. M. (2009). An antifungal agent produced by Streptomyces olive ceicleroticus,

az-sh514. world appl. sci. j. 6, 1495-1505.

5. Fukuchi, N., Futaki, F., Kito, M., Sato, S., Kajiura, T., Ono, Y., et al., (2009). A substance with Antithrombotic Activity and Method for Detecting Glycokallidin. US 7608695.

6. Olano, C., Méndez, C., and Salas, J. A. (2009). Antitumor compounds from Actinomycetes: from gene clusters to new derivatives by combinatorial biosynthesis. Nat. Prod. Rep. 26, 628-660. DOI: $10.1039 / \mathrm{b} 822528 \mathrm{a}$

7. Ser, H. L., Palanisamy, U. D., Yin, W. F., Malek, S. N. A., Chan, K. G., Goh, B. H., et al., (2015). Presence of antioxidative agent, Pyrrolo (1, 2-a) pyrazine-1, 4dione, hexahydro-in newly isolated Streptomyces mangrove soli sp. nov. Front. Microbiol. 6:854. DOI: 10.3389/fmicb.2015.00854

8. Undabarrena, A., Beltrametti, F., Claverías, F. P., González, M., Moore, E. R., Seeger, M., et al., (2016). Exploring the diversity and antimicrobial potential of marine Actinobacteria from the Comau Fjord in Northern Patagonia, Chile. Front. Microbiol. 7: 1135. doi: 10.3389/fmicb.2016.01135

9. Hozzein, W. N., and Goodfellow, M.
(2007). Streptomyces synnematoformans sp. nov., a novel Actinomycete isolated from a dune soil in Egypt. Int. J. Syst. Evol. Microbiol. 57, 2009-2013. DOI: 10.1099/ijs.0.65037-0

10. Awad, H. M., El-Sahed, K., and ElNakkadi, A. (2009). Isolation, screening, and identification of newly isolated soil Streptomyces (Streptomyces sp. NRC-35) for b-Lactamase inhibitor production. World Appl. Sci. J. 7, 637-646.

11. Abd-Alla, M. H., El-Sayed, E. S. A., and Rasmey, A. H. M. (2013). Indole-3-acetic acid (IAA) production by Streptomyces atrovirens isolated from rhizospheric soil in Egypt. J. Biol. Earth Sci. 3, 182-193.

12. Rifaat, H. M., Abd, El Naser, N. H., Helmy, S. M., and Ali, A. M. (2013). Taxonomical studies of certain streptomycetes exhibiting antimicrobial activity isolated from Egyptian soils. J. Cult. Collect. 5, 25-34

13. S. D. Bentley, K. F. Chater, A. M. Cerdeno-T arraga et al.,( 2002). Complete genome sequence of the model actinomycete Streptomyces coelicolorA3(2)," Nature, vol. 417, no. 6885, pp. 141-147, 2002.

14. M. P. Singh, P. J. Petersen, W. J. Weiss et al., (2003). Mannopeptimycins, new cyclic glycopeptide antibiotics produced by Streptomyces hygroscopic us LLAC98: antibacterial and mechanistic activities," Antimicrobial Agents and Chemotherapy, vol. 47 , no. 1, pp. 62-69, 2003.

15. S. A. El-Shatoury, N. S. El-Shenawy, and I. M. AbdElSalam( 2009). Antimicrobial, antitumor and in vivo cytotoxicity of actinomycetes inhabiting marine shellfish. World Journal of Microbiology and Biotechnology, vol. 25, no. 9, pp. 15471555, 2009.

16. Bentley, S. D., Chater, K. F., CerdenoTarraga, A. M., Challis, G. L., Thomson, N. R., James, K. D., et al., (2002). 
Complete genome sequence of the model actinomycete Streptomyces coelicolor A3(2). Nature 417, 141-147. DOI: 10.1038/417141a

17. M. Sosio, E. Bossi, A. Bianchi, and S. Donadio 2000. Multiple peptide synthetase gene clusters in actinomycetes. Molecular and General Genetics, vol. 264, no. 3, pp. 213-221, 2000

18.D. A. Hopwood (2007).Therapeutic treasures from the deep," Nature Chemical Biology, vol. 3, no. 8, pp. 457458, 2007.

19.K. Zengler, A. Parakar, and M. Keller, (2005). New methods to access microbial diversity for small molecule discover, in Natural Product therapeutic Medicine, L. Zhang and A. L. Demain, Eds., pp. 275294, Humana Press, Totowa, NJ, USA, 2005.

20. M. J. Bibb, (2005). Regulation of secondary metabolism in Streptomyces Current Opinion in Microbiology, vol. 8, no. 2, pp. 208-215, 2005.

21. El-Khawaga M. A. and Megahed M. M. M2. (2012). Antibacterial and insecticidal activity of actinomycetes isolated from the sandy soil of (Cairo-Egypt). Egypt. Acad. J. Biology. Sci., 4(1): 53-67

22. Polpass Arul Jose and Solomon Robinson David Jebakumar (2013). Formulation and Statistical Optimization of Culture Medium for Improved Production of Antimicrobial Compound by Streptomyces sp. International Journal of Microbiology Volume 2013, Article ID 526260, 9 pages http://dx.doi.org/10.1155/2013/526260

23. Guillermo Repetto, Ana del Peso, Jorge L Zurita(2008). Neutral Red Uptake Assay for the Estimation of Cell Viability/Cytotoxicity. Nat Protoc. 2008; 3(7):1125-31. DOI: 10.1038/nprot.2008.75.

24- van Keulen, G., and Dyson, P. J. (2014).Production of specialized metabolites by Streptomyces coelicolorA3(2). Adv. Appl. Microbiol. 89, 217-266. DOI: 10.1016/B978-0-12800259-9.00006-8

25. Maxwell, C. A., Hartwig, U. A., Joseph, C. M., and Phillips, D. A. (1989). A chalcone and two related flavonoids released from alfalfa roots induce nod genes of Rhizobium meliloti. Plant Physiol. 91, 842-847. DOI: 10.1104/pp.91. 3.842

26. Singh, K,B.A.D. (2017). Biochemical and molecular studies of the anti phyto pathogenic trait in Actinomycetes (Doctoral dissertation, UASD)

27.Ganesan, P., Reegan, A.D., David, R, H., A, Gandhi, M.R., Paulraj, M.G., Aldhabi, N.A., and Ignacimuthu, S (2017). Antimicrobial activity of some Actinomycetes from western Ghats of Tamil Nadu. India. Alexandria journal of medicine, 53(2), 101-110

28. Tanaka, Y., Kasahara, K., Hirose, Y., Murakami, K., Kugimiya, R., and Ochi, K. (2013).Activation and products of the cryptic secondary metabolite biosynthetic gene clusters by rifampin resistance (rpoB) mutations in actinomycetes. J. Bacteriol. 195, 2959-2970. DOI: 10.1128/JB.00147-13

29. Ikeda, H., Ishikawa, J., Hanamoto, A., Shinose, M., Kikuchi, H., Shiba, T., et al., (2003). Complete genome sequence and comparative analysis of the industrial microorganism Streptomyces avermitilis. Nat. Biotechnol. 21, 526-531. DOI: 10.1038/nbt820

30. Ohnishi, Y., Ishikawa, J., Hara, H., Suzuki, H., Ikenoya, M., Ikeda, H., et al., (2008). Genome sequence of the streptomycin-producing microorganism Streptomyces griseus IFO 13350. J. Bacteriol. 190, 4050-4060. DOI: 10.1128/JB. 00204-08

31. Wakefield, J., Hassan, H. M., Jaspars, M., Ebel, R., and Rateb, M. E. (2017). Dual 
induction of new microbial secondary metabolites by fungal bacterial cocultivation. Front. Microbiol. 8:1284. DOI: $10.3389 /$ fmicb.2017.01284

32. Luo, Y., Huang, H., Liang, J., Wang, M., Lu, L., Shao, Z., et al., (2013). Activation and characterization of a cryptic polycyclic tetramate macrolactam biosynthetic gene cluster. Nat. Commun. 4:894. DOI: $10.1038 /$ ncomms 3894

33. Kalan, L., Gessner, A., Thaker, M. N., Waglechner, N., Zhu, X., Szawiola, A., et al., (2013). A cryptic polyene biosynthetic gene cluster in Streptomyces calvus is expressed upon complementation with a functional bldA gene. Chem. Biol. 20, 1214-1224. DOI: 10.1016/j.chembiol.2013.09.006

34. Gomez-Escribano, J. P., Song, L., Fox, D. J., Yeo, V., Bibb, M. J., and Challis, G. (2012). Structure and biosynthesis of the unusual polyketide alkaloid coelimycin P1, a metabolic product of the cpk gene cluster of Streptomyces coelicolor M145. Chem. Sci. 3, 2716-2720.doi: $10.1039 / \mathrm{c} 2 \mathrm{sc} 20410+\mathrm{j}$

35.Mani Ganesh, Murugan Mohankumar J Food SciTechnol (September 2017). Extraction and identification of bioactive components in Sidacordata (Burm.f.) using gas chromatography-mass spectrometry J Food Sci. Technol (September 2017) 54(10): 3082-3091

36. Prabhanna Banakar and M. Jayaraj (2018). Gc-Ms analysis of bioactive compounds from ethanolic leaf extract of Walthers indica Linn. 2018. And Their Pharmacological Activities IJPSR, 2018; Vol. 9(5): 2005-2010.

37. M. Lakshmi and Bindu R. Nair (2017). Gc-Ms analysis of the chloroform extract of bark of Terminalia travancorensis Wight \& Arn. (Combretaceae) (2017) Ijpsr Vol. 8, Issue 2

38. Subban Murugesan, Ramasamy Vijayakumar, Annamalai Panneerselvam
(2011). Research Journal of Pharmaceutical, Biological and Chemical Sciences Evaluation of Phytochemical Constituents from the Leaves of Memecylonum bellatum Burm.f. Volume 2 Issue 4 Page No. 1145

39. SakshiPainul, Nishant RAI, Navin Kumar (2015). GC-MS analysis of methanolic extract of leaves of Rhododendron campanulatum. Int J Pharm PharmSci, Vol 7, Issue 12, 299-303

40. SorayaGalmánGraíño, Raquel Sendón ID, Julia López Hernández ID and Ana Rodríguez-Bernaldo de Quirós (2018)GC-MS Screening Analysis for the Identification of Potential Migrants in Plastic and Paper-Based Candy Wrappers, 10, 802; doi:10.3390/polym10070802 www.mdpi.com/journal/polymers

41. Mohanad Jawad Kadhim Der Pharma Chemica, (2016) Analysis of bioactive metabolites from Candida albicans using (GC-MS) and evaluation of antibacterial activity. Journal International Journal of Pharmaceutical and Clinical Research Volume8 (19):657-665

42. G. Belakhdar, A. Benjouad1, E.H. Abdennebi J. Mater. Environ (2015). Determination of some bioactive chemical constituents from Thesiumhumile Vahl. J. Mater. Environ. Sci. 6 (10) (2015) 2778-2783.

43. K A Shaheed, N I AlGaraawi, A K Alsultany, Z H Abbas, I K Khshayyish and M T Al khazali (2019). Analysis of bioactive phytochemical compound of (Cyperus iria L.). By using gas chromatography-mass spectrometry 2019International Conference on Agricultural Sciences. Series: Earth and Environmental Science 388 (012064 IOP Publishing DOI:10.1088/17551315/388/1/012064

44. Olena Konovalova, Evgenia Gergel, Vitaliy Herhel (2013). GC-MS Analysis of Bioactive Components of Shepherdia 
argentea (Pursh.) Nutt.from Ukrainian Flora The Pharma Innovation - Journal Vol. 2 No. 6.

5. Velmurugan $G$, Anand $S$ PInternational (2017). GC-MS Analysis of Bioactive Compounds on Ethanolic Leaf Extract of Phyllodium pulchellum L. Desv. (2017). International Journal of Pharmacognosy and Phytochemical Research 2017; 9(1); 114-118 ISSN: 0975-4873

46. Maghdu Nainamohamed Abubacker and Palaniyappan Kamala Devi (2014). In vitro antifungal potentials of bioactive compound oleic acid, 3-(octadecyloxy) propyl ester isolated from Lepidagathis cristata Willd. (Acanthaceae) inflorescence Asian Pac J Trop Med 2014; 7(Suppl 1): S190-S193 DOI: 10.1016/S1995-7645(14)60230-3

47. Madhusudhan KN, Vinayarani G, Moorthy SM, Satish L, Thirupathaiah, Y, Maheshwari C, Prakash HS, Teotia RS, and Sivaprasad V (2019). Isolation, purification and characterization of antibacterial bioactive compounds from Bougainvillea spectabilis Leaf. Journal of Pharmacognosy and Phytochemistry; 8(3): 2668-2673

48. N. Senthilkumar, S. Murugesan, and K. B. Vijayalakshm (2012).GC-MS-MS analysis of Trichilia connaroides (Wight \&Arn.) Bento (Meliaceae): A tree of ethno botanical records Asian Journal of Plant Science and Research, 2 (2):193197.

49. V. Karthikeyan, A. Baskaran, and C. Sebastian Rajasekaran (2016). Gas Chromatography-Mass Spectrometry (GC-MS) Analysis of Ethanolic Extracts of Barleria acuminata Nees. International Journal of Pharmacological ISSN: 22773312.

50. Renu S, MonishaK, Rup L. (2008). Bioactive compounds from marine actinomycetes. Indian J. Microbiol. (48): $410-431$

\section{How to cite this article:}

Tarek R. Elsayed, Diana F. Galil, Mohammed Z. Sedik, Hazem M. M. Hassan, Michael R. Gohar and Mahmoud W. Sadik 2020. Antimicrobial and Anticancer Activities of Actinomycetes Isolated from Egyptian Soils. Int.J.Curr.Microbiol.App.Sci. 9(09): 1689-1700. doi: https://doi.org/10.20546/ijcmas.2020.909.209 